DOCTRINA

\title{
Personas vinculadas al sector agrícola en zonas rurales: migrantes climáticos inminentes
}

\author{
People related to agricultural sector in rural zones: imminent climate migrants
}

\author{
Gustavo Arellano Reyes (iD \\ Investigador independiente, Chile \\ Carolina Silva Santelices (iD) \\ Investigadora independiente, Chile
}

\begin{abstract}
RESUMEN Las migraciones climáticas dentro de un país ocurren por diversos motivos. En este artículo se abordarán aquellas impulsadas por cambios en las condiciones ambientales relacionadas con períodos de sequía, eventualmente acrecentadas por el cambio climático, que afectan a personas ligadas a la agricultura como actividad de subsistencia y/o de desarrollo económico. Se revisarán dos casos relacionados con este fenómeno que presentan características similares, lo que hace interesante su análisis comparativo: uno en la comuna de Monte Patria, región de Coquimbo, Chile, y otro en La Montaña, estado de Guerrero, México. Se analizarán las regulaciones relativas al recurso hídrico, y las políticas públicas asociadas a sequía y cambio climático. Finalmente, se estudiará si existe o no una adecuada respuesta institucional que persiga la prevención, o bien, la mitigación de este tipo de desplazamientos. Esto resulta relevante dado que constituyen procesos de desarrollo continuo y progresivo. Se observa, además, la existencia de personas que se encuentran en una situación de migración inminente por causas similares a los casos analizados.
\end{abstract}

PALABRAS CLAVE Migración interna, agricultores, sequía, cambio climático.

ABSTRACT Climate migrations within a country take place because of various reasons. In this work will been reviewed those that occur due the changes in environmental conditions, specifically related to drought's consequences, increased by climate change, which affect people linked to agriculture as an economic activity or for their own livelihood. Will be review two cases related to this phenomenon that have similar characteristics what makes interesting its comparative analysis: one in the commune of Monte Patria (Coquimbo Region, Chile), and the other in La Montaña (Guerrero State, Mexico). 


\begin{abstract}
Legal regulations related to water resources, public policies related with drought and climate change are going to be analyzed. Finally, it will be studied whether or not there is an adequate institutional response that seeks to prevent or mitigate this type of displacement. The foregoing is relevant because this is a continuous and progressive situation, also observing the existence of people who are in an imminent migration situation, for similar reasons of the analyzed cases.
\end{abstract}

KEYWORDS Internal migration, agriculture, drought, climate change.

\title{
Introducción
}

Existen diversos factores que impulsan a las personas a migrar: trabajo, calidad de vida, acceso a recursos, cambio en condiciones ambientales, entre otros (HoeghGuldberg y otros, 2018), y pueden presentarse de manera conjunta o separada. Para nuestro estudio, sin perjuicio de reconocer la multiplicidad de factores, nos enfocaremos en analizar como causa de un proceso migratorio el cambio en las condiciones ambientales.

En esta línea, las personas que se dedican a actividades económicas dependientes de recursos naturales son susceptibles de experimentar procesos migratorios; por tal motivo, serán sujetos de nuestro estudio las personas de zonas rurales, ligadas a la agricultura como actividad de subsistencia y/o desarrollo económico quienes, ante fenómenos de sequía, eventualmente agravados por el cambio climático —entendiendo a este como un desastre de lenta aparición-, pueden verse imposibilitadas de continuar con dicha actividad y, en consecuencia, por obligación o decisión propia, migrar a otros lugares. Es relevante aclarar que únicamente se abordarán los procesos migratorios internos y quedan excluidos de este artículo los procesos migratorios internacionales.

La metodología de análisis considerará el estudio de lo sucedido en Monte Patria, región de Coquimbo, Chile, y en La Montaña, estado de Guerrero, México.

Se ha optado por estos casos debido a que ambos países, según se expondrá, son particularmente vulnerables a los efectos adversos provocados por el cambio climático; tanto en el caso de Monte Patria como en el de La Montaña, la principal actividad económica de los grupos propensos a la migración es la agricultura; en estos lugares, además, se han observado fenómenos de sequía reciente que, como se explicará, podrían haberse agudizado como consecuencia del cambio climático.

Junto a lo anterior, resulta interesante revisar lo ocurrido en una jurisdicción extranjera, dado que permite contrastar la forma en que dos países enfrentan la situación, sus consecuencias y los mecanismos de adaptación dentro de sus respectivos ordenamientos. Así, es posible levantar como hipótesis que Chile no cuenta con políticas para hacerse cargo de la situación de estos migrantes. No existe mención 
a estos desplazamientos en ningún cuerpo normativo, no se observan medidas mitigatorias ni preventivas asociadas. Esto último es de suma relevancia ya que, como se analizará, actualmente existen otras zonas dentro del país que son susceptibles de experimentar fenómenos similares a los de Monte Patria. Finalmente, cabe destacar que tampoco existe un catastro nacional de carácter gubernamental ni un registro específico que permita observar el desplazamiento al interior del país; tampoco hay estudios respecto a sus causas, lugares de desplazamiento o las consecuencias sociales y económicas que puede generar a nivel familiar, local o regional.

De este modo, en primer lugar, se expondrá el concepto de migrante climático; luego, se procederá a revisar los casos seleccionados; posteriormente, se estudiará la legislación aplicable en cada uno de los países respecto a recursos hídricos y cambio climático, para después exponer las medidas contempladas en cada país respecto a los procesos de migración interna; y finalmente, se proponen recomendaciones en relación con la respuesta institucional existente o inexistente de cada uno de los casos.

Cabe indicar que el trabajo consiste en un análisis multidisciplinario, el que excede un análisis puramente teórico-formal: pretendemos abordar la problemática de la migración interna no únicamente desde un punto de vista jurídico, sino también con perspectivas sociales - estadística, socio-antropológioa, entre otras-, utilizando únicamente fuentes de tipo secundarias y no primarias. El análisis de casos que se desarrolla en este artículo, si bien cuenta con una diversidad de fuentes, no se enfoca en el desarrollo jurisprudencial, sino en un análisis que considera la respuesta institucional proveniente del Poder Ejecutivo, verificando más la existencia y eventual efectividad de políticas públicas que la labor judicial.

\section{Concepto de migrante climático}

La Organización Internacional para las Migraciones (OIM) señala que «los migrantes por motivos ambientales son personas que, debido a cambios repentinos o graduales en el medio ambiente que inciden negativamente en sus condiciones de vida, se ven obligados a -o deciden - abandonar sus viviendas habituales, ya sea de manera temporal o permanente, y se desplazan a otras partes de su propio país o fuera del mismo» (OIM, 2014). En este sentido, quienes se dedican a actividades económicas dependientes de recursos naturales, constituyen, entre otros, potenciales candidatos a migrar por razones climáticas.

En base a lo anterior es posible desprender tres características de la migración por cambio en el clima que merecen un análisis: i) son cambios repentinos o graduales, ii) inciden de manera negativa en las condiciones de vida, y iii) generan como consecuencia la migración forzada o voluntaria.

En primer lugar, los cambios que motivan la migración pueden ser repentinos o graduales. Este artículo se enfocará en los cambios graduales; en particular, aquellos 
que se asocian a la disponibilidad al recurso hídrico para el desarrollo de actividades agrícolas. El cambio climático se define como «un cambio de clima atribuido directa o indirectamente a la actividad humana que altera la composición de la atmósfera mundial y que se suma a la variabilidad natural del clima observada durante períodos de tiempo comparables» (CMNUCC, 1992).

Respecto de los cambios graduales que se abordarán, son relevantes los conceptos de sequía y escasez hídrica. El concepto de sequía puede ser clasificado en diversas categorías (Mishra y Singh, 2016), no obstante, para el análisis que se realiza en este artículo son pertinentes los conceptos de sequía agrícola y socioeconómica. La FAO define sequía agrícola como el porcentaje de años donde los cultivos fallan por falta de humedad (Mishra y Singh, 2010: 206). A nivel nacional, se la define como «el déficit de las precipitaciones o los caudales que afecta la productividad de los cultivos según su estado de desarrollo» (Conaf, 2016: 2019).

A su vez, la sequía socioeconómica ocurre cuando la demanda del bien económico excede la oferta como resultado de un déficit relacionado con el clima en el suministro de agua (Mishra y Singh, 2010: 206). Es lo que se conoce como escasez hídrica (Baeza, 2018: 2). Para efectos de este artículo entenderemos que existe una estrecha relación entre ambos conceptos, aunque el concepto amplio es el de sequía y el más específico el de escasez hídrica.

Una sequía eventualmente puede verse agravada por el cambio climático, dado que según el Intergovernmental Panel on Climate Change (IPCC) se espera que el aumento de las temperaturas en más de $1,5{ }^{\circ} \mathrm{C}$ genere un aumento en la intensidad y frecuencia de las sequías (Hoegh-Guldberg y otros, 2018: 177). En relación con la incidencia negativa que estos fenómenos conllevan, cabe indicar que la sequía es considerada como un proceso de inicio lento que afecta cada vez más la movilidad de la gente, aunque actualmente no existe disponibilidad de datos que permitan cuantificarlo (OIM, 2018: 44) dado que gradualmente disminuye la viabilidad de los sistemas humanos y naturales (Noralene, Shaw y Takeuchi, 2012: 135).

La agricultura es considerada como una de las actividades de mayor susceptibilidad ante los cambios ambientales y juega un rol importante en las formas de vidas rurales (Noralene, Shaw y Takeuchi, 2012: 134). De esta manera, se prevé que el cambio climático afecte la seguridad alimentaria (FAO, 2010a), específicamente del $75 \%$ de la gente cuyos modos de vida dependen de la agricultura (FAO, 2016: 4). En este sentido, algunas investigaciones señalan que el sujeto de estudio de este trabajo representa mayor vulnerabilidad debido a que, en su mayoría, se encuentra en el rango de ingreso más bajo (FAO, 2010a), y no puede acceder, por ejemplo, a sistemas de riego eficaces o a otros mecanismos que permitan una agricultura de subsistencia.

Respecto a las consecuencias que generan estos fenómenos, se observa una decisión de abandonar las viviendas de manera temporal o definitiva para desplazarse a otros lugares dentro del país o fuera de este. Como se mencionó, este trabajo se 
centrará en la migración interna, que corresponde al movimiento de personas de una región a otra en un mismo país. Esta migración puede ser de carácter temporal o permanente (OIM, 2014: 13).

\section{Análisis de casos}

Dentro de los motivos que pueden explicar la elección de México como país de comparación con el caso chileno, destaca que ambos países son particularmente vulnerables a los efectos adversos provocados por el cambio climático (OIM, 2019: 25 y 51). La vulnerabilidad se considera en función de la exposición, sensibilidad y capacidad adaptativa de un país. La exposición se refiere al tipo, grado o naturaleza de las variaciones climáticas a la que un sistema está expuesto. La sensibilidad se refiere al grado en que un sistema resulta afectado positiva o negativamente por la variabilidad o el cambio climático. La capacidad adaptativa hace referencia a los recursos e instituciones que permiten el diseño e implementación de medidas efectivas para la reducción de la vulnerabilidad de la población y de los ecosistemas (Inecc, 2016).

Las condiciones de exposición de ambos países son similares por la ubicación geográfica -zonas propensas a las sequías y desertificación, largos territorios expuestos a tsunamis, entre otros-, aspecto que ambos países cumplen. No obstante, en el caso de México la vulnerabilidad también se expresa a nivel económico y social en el índice de pobreza extrema, que alcanza el 7,4\% (Coneval, 2018). Por el contrario, en Chile esta tasa es de solo el 2,3\% (Ministerio de Desarrollo Social, 2018); además, se le considera como un país con capacidades técnicas e institucionales, y ocupa el primer lugar de la región en muchos de los indicadores relativos a los Objetivos de Desarrollo Sostenible (OIM, 2019: 51). Este aspecto es relevante, dado que Chile, en comparación con México, posee una mejor capacidad, al menos económica, para hacerse cargo de las consecuencias derivadas del cambio climático. No obstante, como se verá, México parece manejar de mejor manera la migración interna.

La Segunda Comunicación Nacional de Cambio Climático de Chile (2011) señaló que nuestro país es altamente vulnerable al fenómeno del cambio climático, ya que cuenta con áreas de borde costero de baja altura, áridas, semiáridas y de bosques, susceptibilidad a desastres naturales, áreas propensas a sequía y desertificación, zonas urbanas con problemas de contaminación atmosférica y ecosistemas montañosos, como las cordilleras de la Costa y de los Andes. Especialmente relevante es la zona central de Chile, que se encuentra dentro de las áreas de alta vulnerabilidad frente a los cambios climáticos. Esto, dado que en el borde costero norte se encuentra el desierto de Atacama que, según se pronostica, aumentará su grado de aridez; las precipitaciones han venido descendiendo entre un 20 y 30 por ciento, y se han observado además cambios en su estacionalidad e intensidad (OIM, 2017: 60). 
México, por su parte, se encuentra ubicado en una «región que es muy susceptible a variaciones significativas en los patrones climáticos y temperaturas» (Ángeles y Gámez, 2013: 35). Influyen tanto su ubicación geográfica, su topografía y su red hidrológica, que lo hacen particularmente frágil a grandes daños por eventos hidrológicos o meteorológicos extremos (WWF, 2010: 2).

El segundo motivo es que ambos países han transitado por períodos de grandes sequías. En el caso de Chile, se ha experimentado un fenómeno denominado megasequía, por tratarse de la más larga de la historia, que ha afectado la mayor área y que ha implicado el desplazamiento de pequeños productores rurales a áreas urbanas o costeras, dejando áreas improductivas (OIM, 2019: 51).

Las sequías no son sucesos aislados en Chile: se conocen diversos episodios de déficit pluviométrico superior al 30\% entre las regiones de Coquimbo y Biobío, entre los años 1940 a 2010. No obstante, a partir del año 2010 se registra un evento, aún en desarrollo, que es el de mayor duración y extensión territorial registrado, y se le ha denominado megasequía (CR2, 2015: 4). La década más cálida de los últimos cien años ha exacerbado el déficit hídrico a través de la evaporación de cuerpos de agua y cultivos; el $25 \%$ de déficit de precipitación se puede atribuir al cambio climático antrópico (CR2, 2015: 2). Datos del boletín de sequía de la Dirección de Meteorología de Chile señalan que en 24 meses (octubre de 2018 a septiembre de 2020), las condiciones secas se observan desde la región de Antofagasta al sur, con características extremas en las regiones de Valparaíso y O'Higgins. ${ }^{1}$ Lo anterior demuestra que la pluviosidad observada durante el año 2020 no resultó relevante en el marco del suceso de sequía observado en el período reciente en Chile.

Lo que explicaría la megasequía serían las condiciones neutras en el Pacífico ecuatorial y la baja oscilación decadal del Pacífico; no obstante, ambos factores no son capaces de explicar por sí solos el fenómeno. El cambio climático antrópico estaría provocando también el déficit hídrico tan marcado y persistente (CR2, 2015: 9).

Los principales impactos de este fenómeno han sido el déficit del promedio en los caudales en los ríos de las regiones de Coquimbo y Valparaíso. Entre 2010 y 2014 alcanzó el 70\%; también se ha reducido el agua almacenada en diversos sistemas algunos como el embalse La Paloma-, aprovechados mayormente para el riego agrícola (CR2, 2015: 12).

Por su parte, México experimentó durante los años 2011 a 2012 una de las peores sequías de las últimas siete décadas (Ortega-Gaucín, 2018: 79), y que afectó a más del $80 \%$ del territorio nacional y causó pérdidas en el sector agropecuario, desabasteciendo del recurso hídrico a las comunidades rurales de las regiones más áridas y vulnerables (Ortega-Gaucín, 2018: 79), además de implicar un gran gasto estatal (Ortega-Gaucín, 2018: 93).

1. Dirección Meteorológica de Chile, Boletín de Sequía núm. 87, disponible en https://bit.ly/3nW3raK. 
Finalmente, cabe señalar que los lugares analizados son un tanto disímiles en términos de magnitud territorial y demográfica: Monte Patria es una pequeña comuna y La Montaña agrupa a diecinueve municipios. No obstante, en ambos la actividad económica principal es la agricultura, lo que permite realizar el análisis migratorio desde un enfoque común.

\section{Monte Patria, Región de Coquimbo, Chile}

Monte Patria se ubica en la Provincia del Limarí, dentro del valle del río del mismo nombre, en la región de Coquimbo, en una zona cordillerana y de altas pendientes.

En relación con su población, revisados los datos obtenidos por el censo poblacional del año 2017 -último válido y disponible-, Monte Patria tiene 30.751 habitantes (15.270 hombres y 15.481 mujeres). Respecto al análisis de población urbana y rural, en los censos de 2002 y 2017 se observa disminución de habitantes en zonas rurales y aumento en zonas urbanas, como muestra la tabla 1.

En lo que refiere a la actividad económica principal de la comuna, prevalecen las asociadas con la agricultura, ganadería, caza y silvicultura, que en su conjunto representan el $69 \%$ del total; le siguen las actividades del rubro de los hoteles y restaurantes, con el $15 \% .{ }^{2}$ De la revisión de los reportes comunales de la Biblioteca del Congreso Nacional y de la información disponible por parte de la Cepal, se observa en la tabla 2 lo siguiente para los años 2009, 2011, 2013 y 2017.3

Sin perjuicio de que este levantamiento de datos no analiza en forma particular la agricultura como actividad económica, sino que la agrupa con otras actividades primarias, entre el 2009 y 2017 se observa una disminución de 8.762 personas dedicada a dichas actividades. En forma menos drástica, para el mismo período se observa una disminución en la mano de obra total de la comuna de 5.328 personas; en este sentido, las actividades primarias, frecuentemente, se desarrollan en zonas rurales y considerando la información de la tabla 1, que demuestra una disminución de población en dichas áreas, es posible plantear que personas dedicadas a actividades primarias, como la agricultura, pueden ser candidatas a un eventual fenómeno migratorio campo-ciudad, motivadas por la búsqueda de empleo.

2. El 13 de enero de 2020 se solicitó información a la Ilustre Municipalidad de Monte Patria (Ley 20.285) respecto al número de habitantes y la principal actividad económica de la comuna. Dicha solicitud fue atendida el 10 de febrero de 2020 y los datos aportados, según informan, provienen del censo poblacional del Instituto Nacional de Estadísticas (INE) del año 2017 y de la base de datos del Servicio de Impuestos Internos (SII) del mismo año.

3. El 13 de enero de 2020 se solicitó información al Instituto Nacional de Estadísticas (Ley 20.285) respecto al número de habitantes que se dedicaban a la agricultura en la comuna de Monte Patria. Dicha solicitud fue atendida el 18 de febrero de 2020 y los datos aportados, según informan, provienen del catastro de la Cepal/Celade Redatam 7, 11 de febrero de 2020. 
Tabla 1. Análisis población urbana y rural años 2002-20017

\begin{tabular}{|lccl|}
\hline Parámetro & Censo 2002 & Censo 2017 & Resultado \\
\hline Hombres zona rural & 8.736 & 7.791 & Disminución de 945 hombres en zonas rurales \\
\hline Hombres zona urbana & 6.615 & 7.479 & Aumento de 864 hombres en zonas urbanas \\
\hline Mujeres zona rural & 8.200 & 7.622 & Disminución de 578 mujeres en zonas rurales \\
\hline Mujeres zona urbana & 6.725 & 7.859 & Aumento de 1.134 mujeres en zonas urbanas \\
\hline
\end{tabular}

Fuente: Censo poblacional año 2017, INE.

Tabla 2. Actividades económicas de la comuna de Monte Patria, años 2009 a 2017.

\begin{tabular}{|lcccc|}
\hline Actividad & 2009 & 2011 & 2013 & 2017 \\
\hline Agricultura, ganadería, caza y silvicultura & 13.771 & 11.315 & 9.085 & 5.009 \\
\hline Total (todo tipo de actividades) & 17.397 & 15.018 & 12.797 & 12.069 \\
\hline
\end{tabular}

Fuente: Reportes Estadísticos de la Biblioteca del Congreso Nacional: Monte Patria, disponible en http://bit.ly/3rB9yng. Y catastro de la (EPAL/ CELADE Redatam 7.

Para la región de Coquimbo, la FAO señala que las personas que habitan zonas rurales consideran que el fenómeno de sequía experimentado en los últimos años ha sido el más importante de las últimas décadas, lo que ha motivado el cuestionamiento de seguir viviendo en dicho territorio. Las principales percepciones locales que levantó el estudio fueron:

Fuertes disminuciones de las fuentes de agua; dificultades o imposibilidad de sembrar hortalizas, trigo, papas y maíz; importante reducción en la producción de frutales; pérdidas económicas asociadas a disminución o ausencia de producción; necesidad de comprar en el mercado los productos que cultivaban destinados al autoconsumo, afectando el presupuesto del hogar y cambiando la conducta alimenticia de las familias (FAO, 2010b: 8).

La situación relatada por el estudio de la FAO es relevante ya que el fenómeno migratorio de esta comuna ocurre en el contexto de la sequía que se vive en la región de Coquimbo. Las comunidades agrícolas pueden ver en la migración una alternativa a la precaria economía de subsistencia cuando se carece del recurso hídrico.

En este contexto, considerando la vulnerabilidad del componente ambiental (índice global de aridez e índice de retención de humedad del suelo), la vulnerabilidad del componente productivo (infraestructura de riego, adopción tecnológica y diversificación productiva) y la vulnerabilidad del componente socioeconómico, el estudio de la FAO concluye que las zonas de secano de la región de Coquimbo son las que 
representan mayor riesgo de afectación por las consecuencias de los fenómenos de sequía, zona en la se ubica Monte Patria, Combarbalá y Río Hurtado.

La OIM señaló que en la comuna de Monte Patria se han desplazado en los últimos diez años una gran cantidad de agricultores junto con sus familias, hacia ciudades como La Serena, Ovalle y Copiapó, en forma permanente, y muchos hombres se trasladaron al norte a trabajar en la minería, migrando de lugar y de actividad económica. En el estudio se observan dos tipos de desplazamientos: permanentes y temporales; estos últimos admiten tres modalidades: personas que migran y luego de cada sequía vuelven a su hogar y al trabajo de la agricultura; los que se alternan, primero se traslada el hombre, retorna y luego se va la mujer; y los casos en que se va el hombre y la mujer, quedando los hijos al cuidado de un tercero, como abuelos o tíos (OIM, 2017: 87). Por su parte, la FAO señala que las migraciones observadas corresponden a un impacto de la sequía y que pueden ser clasificadas como una estrategia adaptativa (FAO, 2010b: 67).

Según el estudio de la OIM, las principales causas de la migración vinculada a la agricultura son: la disminución de la disponibilidad del agua con motivo del cambio climático; la privatización de los derechos de agua de la zona, en desmedro de pequeños agricultores; y el mal uso del recurso hídrico por parte de las compañías mineras de la región (OIM, 2017: 105-109).

El estudio de la OIM concluye que las frecuentes sequías en la comuna, combinadas con carencias importantes en la gestión del agua, constituyen una de las principales razones que inducen a la población a migrar hacia otras regiones del país, de forma temporal y/o permanente (OIM, 2017: 16). Las personas afectadas por la falta de trabajo han emigrado principalmente hacia el norte de Chile: los hombres para trabajar en el sector minero y las mujeres en servicios. Además, el proceso migratorio es continuo, se encuentra en desarrollo y es probable que se perpetúe en el tiempo, considerando el fenómeno de sequía que existe en Chile.

\section{La Montaña, Guerrero, México}

La Montaña es un conjunto de diecinueve municipios localizado en el Estado de Guerrero, donde habitan aproximadamente 355.000 personas (INEGI, 2011: 182). Los Índices de Marginación por Entidad Federativa y Municipio de 2010 indican que en Guerrero el 16,8\% de la población de quince o más años es analfabeta y que el 31,6\% no terminó la primaria (Tlanichollan Centro de Derechos Humanos La Montaña, 2013: 17). La principal actividad económica es la agricultura temporal de autoconsumo, específicamente maíz, frijol y calabaza.

En comparación a los datos existentes para Monte Patria, en México existen datos específicos asociados a procesos de migración interna. Existen datos censales, respecto de aquella población que ha emigrado desde su tierra de origen; del total del país, 
Guerrero acumula el 3,6\% de la población que se encontraría en esta situación. En relación con el dato de los migrantes internos recientes por entidad federativa expulsora entre 2000 y 2010, Guerrero aportó el 3,3\% del total nacional (INEGI, 2010: 60). El saldo neto migratorio interno reciente por entidad federativa del año 2010 muestra que Guerrero presenta una pérdida de entre 30 y 60 mil habitantes (INEGI, 2010: 62).

Adicionalmente, se considera la estructura de edad y sexo de los migrantes internos recientes; la tendencia indica que la población más propensa a migrar se ubica entre los 15 a los 59 años -67,8\% para 2010- existiendo una leve tendencia a la predominancia del sexo femenino por sobre el masculino (INEGI, 2013: 55).

Según datos publicados por la Secretaría de Desarrollo Social en México (Sedesol), en el año 2015 se reportó que los jornaleros agrícolas ${ }^{4}$ migrantes originarios de otras entidades federativas fueron 680.000, en 2016 descendieron a 221.00o y en 2017 dicha población alcanzó a 216.00o; la baja registrada podría obedecer a criterios metodológicos de cifras o a subregistros de estimaciones o datos existentes (CESOP, 2019: 4).

La Ciudad de México y Guerrero siempre han aparecido entre las tres entidades con mayor tasa neta negativa de migración en el periodo de referencia. Sin embargo, por primera vez en la historia reciente, en 2015 el estado de Guerrero superó la tasa neta negativa de la Ciudad de México: 5,2 versus 5,0 (Conapo, 2015b: 16).

Guerrero se caracteriza por ser un proveedor de jornaleros agrícolas, especialmente para Sinaloa (Granados Alcantar y Quezada Ramírez, 2018: 351): en 2013, se concentró aquí el 90\% de la población jornalera proveniente de la región Montaña y Costa de Guerrero (Tlanichollan, 2013: 29).

Se estima que la migración en este estado ha sido más bien una estrategia de sobrevivencia. Asimismo, se ha constatado que inicialmente los procesos migratorios eran marcadamente masculinos; no obstante, en la actualidad son de carácter familiar: el $81 \%$ de migrantes tienen un vínculo de pareja, y se emplea prácticamente todo el grupo familiar en labores agrícolas (Tlanichollan, 2013: 30 y 50). En este contexto, dentro de las consecuencias derivadas de la migración - ex post-, se observan fenómenos de discriminación, subordinación y violencia que sufre la mujer migrante, situación que a la fecha es invisibilizada por los órganos del estado (Tlanichollan, 2013: 25).

4. Jornalero agrícola: «Persona que percibe un salario por su fuerza de trabajo, en una actividad propia del campo dentro de un proceso productivo. En la mayoría de los casos, el salario otorgado es por día y se denomina jornal. En materia laboral no se distingue entre el trabajador del campo o la ciudad, ya que la connotación jurídica ha quedado inserta en el concepto de salario. Al respecto, el artículo 279 de la Ley Federal del Trabajo determina las actividades desarrolladas por los jornaleros agrícolas, bajo el rubro de trabajos especiales» (Gamboa y Gutiérrez, 2015: 6). 
Otro aspecto relevante de la migración del estado de Guerrero es que presenta un alto índice de hablantes de lengua nativa, cuyos migrantes se dirigen principalmente al estado de Sinaloa, de manera que Guerrero aporta un 30,2\% de la población migrante nativa (Granados Alcantar y Quezada Ramírez, 2018: 351).

Específicamente, Tlapa de Comonfort, municipio de La Montaña, es considerado un lugar con alta vulnerabilidad a la sequía: ${ }^{5}$ presenta altos índices de migración hacia otros lugares, como Sinaloa, Jalisco, Baja California y Chihuahua.

Una característica de la migración interna es que se emprende con la familia, dado que así todos los miembros pueden desempeñar funciones productivas en los lugares a los que se trasladan (Morales Jiménez y otros, 2018: 91).

Según entrevistas realizadas a los migrantes de Tlapa, una de las razones por las que la producción de maíz es tan baja - grano de maíz por hectárea- se debería a la falta del recurso hídrico; este factor es considerado como el principal detonante de la decisión de migrar (Morales Jiménez y otros, 2018: 90). Los migrantes en esta zona en su mayoría $(96,3 \%)$ son temporales, regresando una vez al año a su lugar de origen (Morales Jiménez y otros, 2018: 90).

Luego de haber revisado lo sucedido en Monte Patria y en La Montaña, se observa, de modo preliminar, que:

- ambos lugares se han caracterizado por presentar flujos migratorios al interior de sus respectivos países como estrategia de sobrevivencia o estrategia de adaptación;

- entre los motivos para migrar, se encuentra la falta del recurso hídrico para el éxito de las cosechas;

- en ambos, el proceso migratorio se ha desarrollado en forma continua y progresiva;

- en el caso de Monte Patria, destaca la migración masculina hacia el norte del país para emplearse en el sector minero;

- en el caso de La Montaña, la población migrante se mueve con la familia y se dirige principalmente al estado de Sinaloa para emplearse como jornaleros agrícolas;

- en el sujeto migrante en México predomina la pertenencia a alguna etnia originaria, situación que no se observa en el caso chileno, o al menos no ha sido registrado.

5. Comisión Nacional del Agua (Conagua), 2020, Programa Nacional contra la sequía: Monitoreo de la sequía. Disponible en http://bit.ly/38qFD8g. 


\section{Análisis jurídico}

Luego de revisar las principales características de los procesos migratorios seleccionados, resulta relevante referirse a los aspectos normativos asociados a los recursos hídricos y al cambio climático en ambas jurisdicciones.

\section{Estatuto jurídico de las aguas}

El paradigma regulatorio sobre este tema es muy diverso en ambos países. En Chile no se reconoce el derecho humano al agua a nivel constitucional, sino que es considerado un bien de consumo susceptible de ser apropiado por particulares mediante derechos de aprovechamiento, con las únicas limitantes de considerar la disponibilidad material del recurso en los ecosistemas y la no afectación de derechos de terceros legalmente constituidos.

México, en cambio, reconoce desde el año 2012 a nivel constitucional el derecho humano al agua para consumo personal y doméstico. Por su parte, la Ley de Aguas Nacionales, vigente desde el año 1992, establece un sistema de concesión — para personas físicas o morales de carácter público o privado- y asignación — para municipios, estados o el Distrito Federal- para la explotación, uso o aprovechamiento de las aguas nacionales.

En el caso de Chile, el artículo 19 número 24 de la Constitución, vinculado al del derecho de propiedad, dispone que «los derechos de los particulares sobre las aguas, reconocidos o constituidos en conformidad a la ley, otorgarán a sus titulares la propiedad sobre ellos». Sin embargo, el artículo 589 del Código Civil señala que el dominio de dichos bienes corresponde a la nación toda y su uso pertenece igualmente a todos los habitantes. Más adelante, el artículo 595 del mismo cuerpo legal establece que «todas las aguas son bienes nacionales de uso público». El Código de Aguas, en su artículo 5, en armonía con lo dispuesto en el artículo 595 citado, regula que las aguas son bienes nacionales de uso público, pero agrega que «se otorga a los particulares el derecho de aprovechamiento de ellas, en conformidad a las disposiciones del presente Código».

En términos generales, el marco jurídico chileno de las aguas reconoce el derecho de aprovechamiento como un derecho real que recae sobre las aguas y otorga a su titular el uso, goce y disposición, junto con todos los medios necesarios para ejercerlos.

Parte de la doctrina señala que el sistema vigente del derecho de aguas, en cuanto al acceso y aprovechamiento, implica solo la existencia de una prohibición general previa a extraer aguas, bajo la reserva de obtener una «concesión de aguas», que constituye un acto administrativo de naturaleza autorizatoria, con una discrecionalidad reducida (Vergara, 2011: 76). Además, se ha dicho que: 
El hecho que sea el Código de Aguas - y no la Constitución - el que disponga que las aguas son un bien nacional de uso público, para luego regular el otorgamiento de derechos de aprovechamiento de aguas, unido a la visión economicista con que se ha abordado el uso y gestión del agua, fueron generando una interpretación normativa en la que se prioriza el aseguramiento del derecho de propiedad sobre el derecho de aprovechamiento, relegando a un segundo plano otros usos, incluso el humano (Gutiérrez Oliva, 2019: 8).

A similar conclusión arriba el Centro de Ciencia del Clima y la Resiliencia (CR2), al señalar que «la legislación de aguas vigente busca asegurar el abastecimiento con fines productivos y de consumo de los recursos hídricos» (CR2, 2015: 20).

Así, se tiene que el agua se regula como un bien nacional de uso público y como un bien económico, lo que promueve la regulación del uso y acceso a través de una especie de «mercado del agua», donde prima la dinámica de la oferta y la demanda, por encima de las necesidades de la población y los debidos resguardos ambientales que se requieran para asegurar su existencia (Larraín Ruiz-Tagle, 2006: 2). En este sentido, «existen hoy personas naturales y jurídicas que se dedican a acumular derechos de aprovechamiento para especular con ellos en un mercado donde el bien se hace cada vez más escaso [...] en vez de fomentar la libre competencia como medida de eficiencia de uso en el recurso» (Larraín Ruiz-Tagle, 2006: 2).

Sin perjuicio de lo anterior, el Código de Aguas regula ciertas limitaciones para el otorgamiento de derechos de aprovechamiento. Se contemplan, entre otras, las áreas de restricción —-donde solo se otorgan derechos provisorios-, áreas de prohibición — donde no se pueden otorgar derechos_- declaración de zonas de agotamiento donde no hay disponibilidad para constituir nuevos derechos de aprovechamiento-, entre otros.

Por su parte, la Constitución Política de los Estados Unidos Mexicanos reconoce en su artículo 4:

Toda persona tiene derecho al acceso, disposición y saneamiento de agua para consumo personal y doméstico en forma suficiente, salubre, aceptable y asequible. El Estado garantizará este derecho y la ley definirá las bases, apoyos y modalidades para el acceso y uso equitativo y sustentable de los recursos hídricos, estableciendo la participación de la Federación, las entidades federativas y los municipios, así como la participación de la ciudadanía para la consecución de dichos fines.

Es posible destacar que el Estado mexicano, al ser garante de dicho derecho, se ve obligado a:

a) abstenerse de obstaculizar directa o indirectamente su goce - obligación de respetar-; b) impedir a terceros injerencia en su disfrute - obligación de proteger-; y c) adoptar medidas legislativas administrativas o presupuestarias, judiciales, pro- 
moción y de otra índole adecuadas para hacerlo plenamente efectivo —obligación de realizar- ${ }^{6}$

Igualmente, cuando los privados prestan el servicio de abastecimiento de agua deben cumplir dichos deberes.

El derecho al agua no solo se contempla para uso personal en México, sino también como un «beneficio colectivo que debe basarse en criterios de solidaridad, cooperación mutua, equidad y en condiciones dignas».7 Ahora bien, la Ley de Aguas Nacionales de México, al igual que en la legislación chilena, instaura un sistema de concesiones y asignaciones. $\mathrm{Al}$ respecto, su artículo 20 dice:

De conformidad con el carácter público del recurso hídrico, la explotación, uso o aprovechamiento de las aguas nacionales se realizará mediante concesión o asignación otorgada por el Ejecutivo Federal a través de la Comisión por medio de los Organismos de Cuenca, o directamente por ésta cuando así le competa, de acuerdo con las reglas y condiciones que dispone la presente Ley y sus reglamentos.

A su vez, el artículo 22 señala que las concesiones y asignaciones establecerán las condiciones a las cuales estará sujeta la extracción de volúmenes ante sequías y otros fenómenos.

De lo anterior se puede concluir que tal como en Chile, en México, sin perjuicio que la Constitución reconozca el derecho humano al agua, existe la posibilidad de, cumpliendo ciertos requisitos, apropiarse del derecho de explotar, usar o aprovechar las aguas. En relación con esta dualidad, cabe indicar que el artículo 3 transitorio de la modificación constitucional del 8 de febrero de 2012 que incorporó a la Constitución mexicana el derecho humano al agua, estableció que se dictaría en un plazo de 360 días una Ley General de Aguas. No obstante, habiéndose cumplido dicho plazo, aún no ha sido dictada. En la actualidad se observan dos posturas en relación con el eventual estatuto regulatorio: una privada, que aboga por una regularización del recurso con mayor énfasis en aspectos económicos, y otra desde la sociedad civil, que aboga por su democratización, poniendo el foco en la categoría de derecho humano accesible y de calidad para la población (Agua para Todos, 2020). Esta última propone una distinción entre agricultura de sobrevivencia (propone favorecer los sistemas de riego campesino con exención de impuestos y subsidios de bombeo) y agricultura industrial (que pagaría derechos) (Agua para Todos, 2020).

6. Gaceta del Semanario Judicial de la Federación, mayo de 2018, número de registro 2016922, disponible en https://bit.ly/38oXjsh.

7. Gaceta del Semanario Judicial de la Federación, septiembre de 2012, número de registro 2001560, disponible en https://bit.ly/3mYaj63. 


\section{Medidas legales ante fenómenos de sequía}

En relación con los fenómenos de sequía, los estatutos jurídicos de ambos países establecen la posibilidad de decretar, bajo ciertas circunstancias y por un período de tiempo, algunas limitantes en el uso y aprovechamiento del recurso hídrico.

En el caso de Chile, el artículo 314 del Código de Aguas establece que el Presidente de la República, a petición o con informe de la Dirección General de Aguas (DGA), y ante fenómenos de extraordinaria sequía, podrá declarar zonas de escasez por períodos máximos de seis meses, no prorrogables. Además, señala que, declarada la zona de escasez, y no habiendo acuerdo de los usuarios para redistribuir las aguas, la DGA podrá hacerlo respecto de las aguas disponibles en las fuentes naturales, para reducir al mínimo los daños generales derivados de la sequía. Guillof (2013: 247) sostiene que dichas declaraciones no son idóneas para el fin propuesto, dado que se establece la reasignación proporcional de los derechos existentes, lo que podría implicar que el Estado tenga que indemnizar a aquellos titulares que no puedan hacer uso completo de sus derechos, por lo que un error en dicha distribución podría resultar muy costoso para el Estado. También llama la atención que la DGA podrá autorizar extracciones de aguas superficiales o subterráneas desde cualquier punto sin necesidad de constituir derechos de aprovechamiento de aguas y sin la limitación asociada al caudal ecológico mínimo.

Dentro de las indicaciones sustitutivas realizadas por el ejecutivo al proyecto de ley que reforma el Código de Aguas, ${ }^{8}$ se ha señalado respecto del artículo 314 lo siguiente:

- La declaración de escasez la debe realizar el Presidente previa petición e informe de la Dirección General de Aguas (DGA). La disposición actual no establece que el referido informe sea obligatorio, por lo que podría darse el caso de que exista una declaratoria sin fundamentación.

- La definición de las épocas de sequía extraordinaria se someterá a la revisión de un panel de expertos en recursos hídricos y se basará en criterios objetivos. Lo anterior, a diferencia de la actual regulación, permitiría asegurar que la declaratoria cuente con una justificación técnica.

- La duración de la medida quedará supeditada a condiciones establecidas en el propio decreto que la declare, junto con el plazo en que debe ser revisada. La actual redacción establece que la declaratoria podrá ser de seis meses no prorrogables, período que, probablemente, no es suficiente para gestionar adecuadamente un recurso que se ve afectado por fenómenos como la sequía.

8. Indicación sustitutiva al proyecto de Ley que reforma el Decreto con Fuerza de Ley 1.122, Boletín 7.543-12, aún en tramitación. 
- Las extracciones de agua que se autoricen, sin contar con derechos de aprovechamiento y sin la limitación del caudal ecológico mínimo, deberán realizarse privilegiando el uso de las aguas para consumo humano. La indicación establece de esta forma como objeto de protección el consumo humano del agua. En la actualidad se otorga la facultad a la Dirección, sin mayores restricciones y/o condiciones, para autorizar extracciones de agua superficiales o subterráneas, no priorizando ningún tipo de uso, lo que en la práctica podría generar el riesgo de dejar a una porción de población sin abastecimiento de este recurso, ya sea para su subsistencia o para el desarrollo de sus actividades económicas.

El análisis y eventual modificación del artículo 314 del Código de Aguas es relevante, porque en la legislación chilena el Estado únicamente tiene facultad para asignar derechos de aprovechamiento de las aguas y las organizaciones de usuarios son las encargadas de implementar su distribución. Sin embargo, según prescribe el actual texto del artículo 314, si no hay acuerdo de los usuarios, el Estado - por medio de la Dirección- puede hacer uso de la facultad de distribución, en el contexto de una declaratoria de escasez.

Por su parte, el título quinto de la Ley de Aguas Nacionales de México señala la posibilidad de establecer zonas reglamentadas, de veda o de reserva. El artículo 38 dispone:

El Ejecutivo Federal, previos los estudios técnicos que al efecto se elaboren y publiquen, y considerando los programas nacionales hídricos y por cuenca hidrológica y las necesidades del ordenamiento territorial nacional, regional y local, así como lo dispuesto en los artículos 6 y 7 de la presente Ley, podrá decretar el establecimiento de zonas reglamentadas, zonas de veda o declarar la reserva de aguas. Adicionalmente, el Ejecutivo Federal podrá declarar como zonas de desastre aquellas cuencas hidrológicas o regiones hidrológicas que, por sus circunstancias naturales $o$ causadas por el hombre, presenten o puedan presentar riesgos irreversibles a algún ecosistema.

Posteriormente, el artículo 39 inciso final señala que, en casos de sequías extraordinarias, sobreexplotación grave de acuíferos o condiciones de necesidad o urgencia por causa de fuerza mayor, el Ejecutivo Federal adoptará medidas necesarias para controlar la explotación, uso o aprovechamiento de las aguas nacionales, las mismas que se establecerán al emitir el decreto correspondiente para el establecimiento de zonas reglamentadas. Asimismo, el artículo 39 bis define los casos en que el Ejecutivo Federal puede decretar zonas de veda para la explotación, ante situaciones como sequía o escasez extrema.

Respecto al inicio del período de sequía en México, este se declara mediante un «Acuerdo de carácter general de inicio de emergencia por ocurrencia de sequía severa, extrema o excepcional en cuencas» — se consultó el Acuerdo del año 2018-, dic- 
tado por la Comisión Nacional del Agua (Conagua) para un año determinado, con base en los análisis de sequía que se realicen según el monitor de sequía en los casos en que la esta sea severa, extrema o excepcional y se considera para estos efectos, los resultados por cuencas.

Conagua debe garantizar el abastecimiento doméstico y público urbano del recurso hídrico para lo cual podrá ejecutar las medidas transitorias (Ley de Aguas Nacionales, 1992, artículo 9 letra L y 13 bis 4) concertadas con los representantes de los usuarios en los Consejos de Cuenca, en aquellas zonas que presenten condiciones de sequía severa, extrema o excepcional según los programas de medidas preventivas y de mitigación que se dictan para cada cuenca afectada; pudiendo limitarse transitoriamente los derechos de aguas existentes.

A su vez, los titulares de concesiones para la explotación, uso y aprovechamiento podrán avisar a la autoridad cuando proporcionen a terceros en forma provisional el uso parcial o total de las aguas concesionadas, y/o cedan o transmitan temporalmente sus derechos y solicitar la interrupción de la caducidad de los volúmenes concesionados que no utilicen en tanto persista la emergencia. ${ }^{9}$

Las disposiciones referidas precedentemente, a diferencia del actual texto del artículo 314 del Código de Aguas de Chile:

- Consideran como requisito la existencia de informes técnicos que fundamenten y/o justifiquen la declaración de una Zona Reglamentada, de Veda o de Reserva.

- Requieren, además, que se consideren los programas nacionales hídricos y por cuenca hidrológica y las necesidades del ordenamiento territorial nacionales, regionales y locales.

- No establecen una limitación temporal para la declaración.

- Establecen la posibilidad de declarar como zona de desastre a aquellas cuencas o regiones hidrológicas que por sus circunstancias naturales o antrópicas presenten, o puedan presentar riesgos irreversibles a algún ecosistema.

- Establecen un orden de prelación en los usos del agua, privilegiando el uso doméstico y público urbano.

- Se establecen posibilidades de gestión del agua para los concesionarios y para los Consejos de Cuenca, es decir, se trata de una emergencia que se gestiona de manera participativa con varios actores sociales.

9. Acuerdo de carácter general de inicio de emergencia por ocurrencia de sequía severa, extrema o excepcional en cuencas para el año 2018, publicado en el Diario Oficial de la Federación el 9 de julio de 2018. 
Para el caso de Chile, se observa, además, que el Presidente, en función de las atribuciones que le confiere el número 6 del artículo 32 de la Constitución Política de la República, frente a fenómenos de sequía puede declarar a las áreas afectadas como zona de catástrofe y, de esta forma, implementar medidas de excepción que permitan enfrentar de manera más eficiente y eficaz los efectos adversos de una catástrofe. Lo anterior se realiza a través de la coordinación y ejecución de programas de recuperación a cargo de los intendentes regionales. ${ }^{10}$

En lo que refiere a los usos agrícolas del agua, la Ley de Aguas Nacionales de México, a diferencia del Código de Aguas de Chile, reconoce el uso agrícola, específicamente el riesgo (Ley de Aguas Nacionales, artículo 3, numeral LIII). Adicionalmente, si bien el artículo constitucional no reconoce expresamente el derecho al agua para otros usos, como el agrícola, debe entenderse en dicha amplitud, dada la estrecha relación que existe entre este derecho y otros derechos humanos, como los relativos a la alimentación y a la salud. Debe entenderse dicho artículo a la luz del Pacto Internacional de Derechos Económicos, Sociales y Culturales. ${ }^{11}$ Regulación asociada al cambio climático

Respecto la regulación para enfrentar el cambio climático, en Chile no existe un cuerpo normativo que lo regule de manera orgánica. $\mathrm{El} 13$ de enero de 2020, mediante el Boletín 13191-12, ingresó al Congreso el Proyecto de Ley que fija la «Ley Marco para el Cambio Climático», el cual no contempla medidas específicas en relación con los desplazamientos poblacionales motivados por el cambio climático. No obstante, en relación con a la estrategia climática de largo plazo, dentro de los aspectos fundamentales considera lineamientos para que las medidas de adaptación pongan especial énfasis en la sostenibilidad ambiental en el uso del agua frente a amenazas y riesgos asociados a sequías (artículo 5, letra $\mathrm{f}$ ) y se señala que el Ministerio de Obras Públicas, a través de la DGA, elaborará un Plan Estratégico de Recursos Hídricos en Cuencas, el cual deberá considerar medidas concretas para hacer frente a los efectos adversos derivados del cambio climático tales como sequías (artículo 12, letra e).

En México, la Ley de Cambio Climático fue publicada el 6 de junio de 2012, y contempla diversas acciones de adaptación:

- Respecto al recurso hídrico, se indican acciones a ser desarrolladas por entidades gubernamentales, como diagnosticar volúmenes disponibles de agua y su distribución territorial (artículo 30, numeral x) y promover el aprovechamien-

10. Ejemplos de lo anterior: Decreto 234, de 2012, declara como afectadas por catástrofe de sequía las comunas de la La Ligua, Cabildo y Petorca; Decreto 856, de 2012, declara como afectadas por catástrofe de sequía la provincia del Limarí y las comunas de Salamanca, Illapel, Canela, Andacollo y La Higuera.

11. Suscrito y ratificado por México y publicado en el Diario Oficial de la Federación el 12 de mayo de 1981. Véase Gaceta del Semanario Judicial de la Federación, julio de 2015, número de registro 2009628, disponible en https://bit.ly/3rBb34V. 
to sustentable de las fuentes superficiales y subterráneas de agua (artículo 30, numeral xi), entre otras.

- Respecto de la agricultura, contempla la obligación de formular, regular, dirigir e instrumentar acciones adaptativas al cambio climático para actividades primarias. Además, establece que será tarea de entidades gubernamentales fomentar prácticas de agricultura sustentable, así como sistemas de alerta temprana sobre temperaturas anormales (artículo 30, numeral xii).

- Respecto de la situación de los migrantes, menciona que las políticas nacionales adaptativas deben contemplar expresamente la situación de los desplazados internos provocados por fenómenos asociados al cambio climático (artículo 28, numeral vii). Asimismo, establece que las entidades gubernamentales deberán prevenir y atender el posible desplazamiento interno de personas provocadas por fenómenos relacionados con el cambio climático (artículo 30, numeral ii).

\section{Respuestas institucionales a la migración interna}

A continuación, se revisarán las respuestas institucionales ante el fenómeno de migración interna en ambos países, así como otras medidas implementadas para los fenómenos asociados a la sequía o aspectos hídricos relacionados con la actividad de la agricultura.

\section{Respuesta institucional en Chile}

A nivel institucional, en Chile no se observa la existencia de normativas, planes, programas o políticas que regulen, analicen, estudien y/o implementen medidas preventivas para procesos de migración interna, que hayan sido motivadas por fenómenos climáticos y/o ambientales. Lo anterior es relevante dado que la situación migratoria observada en Monte Patria corresponde a un proceso de desarrollo continuo y progresivo que puede intensificarse en la medida en que el fenómeno de sequía se perpetúe en el tiempo. Además, a la fecha se han dictado nueve decretos que declaran zonas de escasez hídrica en las regiones de Coquimbo, Valparaíso y Metropolitana, cuya fecha de caducidad corresponde al año 2019, y junto a los decretos que declaran zonas de catástrofe por sequía en las mismas regiones. Podría presumirse que existen grupos de población rural dedicada a la agricultura susceptible de protagonizar procesos migratorios similares al analizado.

A mayor abundamiento, el Programa de Acción Nacional de Lucha contra la Desertificación, la Degradación de las Tierras y la Sequía (PANCD) señala: 
El fenómeno de sequía a nivel nacional expresado en términos de superficie afecta a aproximadamente el $72 \%$ de las tierras del país, lo que corresponde [...] a $55 \mathrm{mi}$ llones de hectáreas. La población bajo algún riesgo de sequía asciende aproximadamente a 16 millones de habitantes, lo que equivale al 90\% de los habitantes del país, distribuidos en 317 de las 345 comunas del país (Conaf, 2016b: 12).

Al respecto, la Estrategia Nacional de Cambio Climático y Recursos Vegetacionales 2017-2025 agrega:

Las regiones que poseen las mayores superficies de tierras afectadas por sequía grave están en primer lugar la Región de Coquimbo, con aproximadamente 3,9 millones de hectáreas, seguida por la Región de Atacama, con 1,8 millones de hectáreas (Conaf, 2016b: 17).

A pesar de la existencia de localidades en distintas regiones del país susceptibles de experimentar estos fenómenos, Chile solo aborda las migraciones internacionales. No es posible observar estudios que analicen en profundidad lo que ocurre con la migración interna. Tampoco hay estudios que identifiquen y analicen las causas y magnitudes de fenómenos migratorios. No es posible, por lo tanto, definir cuántas personas se ven expuestas a situaciones similares a las vividas en Monte Patria.

El Ministerio de Desarrollo Social, tomando en consideración los datos de la Encuesta de Caracterización Socioeconómica en sus versiones 2009, 2011, 2013 y 2015, hizo un análisis descriptivo con énfasis en la caracterización socioeconómica de los migrantes y en el movimiento de población entre regiones (Ministerio de Desarrollo Social, 2018). Sin embargo, no se detuvo en el análisis de la población agrícola proveniente de zonas rurales.

Respecto a las motivaciones del proceso migratorio interno, el Ministerio de Desarrollo Social solo lo esboza en términos amplios considerando las características sociodemográficas de los migrantes, señalando que la mayoría se ha dirigido a zonas urbanas y que, en general, predominan las mujeres (Ministerio de Desarrollo Social, 2018: 8). Esto coincide con lo señalado por el estudio de la OIM.

Ante casos de sequía similares a los observados en Monte Patria, la FAO hizo un análisis en torno a la gestión pública y privada del riesgo. Respecto de la gestión pública, determinó que existe una actuación enfocada en la respuesta del fenómeno, como «bonos de varios tipos, desparasitaciones de emergencia, reorientación de programas, facilidades para el endeudamiento, infraestructura para el abastecimiento de agua y entrega de canastas familiares» (FAO, 2010b: 8). Un ejemplo de lo anterior se observa en alguna de las acciones de excepción que establecen los decretos que declaran zonas de catástrofe por sequía, donde se ha establecido, para pequeños y medianos agricultores, medidas de alivio tributario, así como facilidades para el pago 
de patentes por no uso de derechos de aprovechamiento de agua. ${ }^{12}$ No obstante, no define medidas directas para los fenómenos migratorios que puedan generarse como consecuencia de dicha catástrofe. En cuanto a la gestión privada, el estudio concluye que los agricultores desarrollan pocas acciones, por lo que sus estrategias de respuesta son limitadas y apuntan a la sobrevivencia del sistema agrícola.

En este orden, cabe señalar que, mediante el Decreto 152 de 2016 del Ministerio del Interior y Seguridad Pública, se aprobó la Política Nacional para la Gestión del Riesgo de Desastres: el texto considera a la sequía como uno de los tipos de desastres y un detonante de emergencias agrícolas, sin embargo, no aborda dentro de sus consecuencias a los agricultores con necesidad de migrar.

En atención a la gestión de riesgos y a las consecuencias derivadas de la sequía, dentro de las que se listan los procesos migratorios en Chile, cabe mencionar al PANCD, que persigue identificar, prevenir y controlar las causas que provocan fenómenos como la desertificación y la sequía. Dentro de las acciones que propone, destaca la de contribuir al fomento de la gestión sustentable de la tierra. Uno de los objetos que motivan dicha acción es la reducción de la migración forzada (Conaf, 2016b: 161). Se reconoce que la desertificación acelerada por el cambio climático precipita procesos migratorios debido a la degradación de ecosistemas y cambios de uso de suelo (Conaf, 2016b: 27-28).

Respecto a la regulación del recurso hídrico en Chile, fue posible observar ciertas deficiencias que no permiten asegurar la subsistencia de la actividad agrícola frente a fenómenos como la sequía, lo que en definitiva puede fomentar la decisión de migrar de todos aquellos que dependen de dicha actividad. Dentro de los criterios para otorgar derechos de aprovechamiento de aguas, no hay consideraciones sobre los usos del territorio ni tampoco se aprecia un principio de equilibrio entre grandes iniciativas, como la minería, y pequeños actores, como las comunidades rurales agrícolas.

En relación con las temáticas propias de cambio climático, en Chile existe una Estrategia Nacional de Cambio Climático y Recursos Vegetacionales 2017-2025, aprobado por el Consejo Directivo de la Corporación Nacional Forestal (en octubre de 2016) y por el Consejo de Ministros para la Sustentabilidad (en noviembre de 2016). Dentro de los impactos socioambientales del cambio climático, dicha estrategia considera la emigración hacia sectores urbanos y el desempleo y disminución de los ingresos rurales campesinos, ambos impactos que se relacionan con nuestro estudio. No obstante, dentro de las medidas que propone, solo se observan dos medidas asociadas en forma indirecta a estos impactos: la implementación de tecnología para fomentar la agricultura familiar (programa de investigación agropecuaria) y el fomento agrícola desde una perspectiva de sustentabilidad (programa de ordenación

12. Ejemplo de lo indicado es el Decreto 1.523, de 2014, que autoriza medidas de alivio tributario para los pequeños y medianos agricultores de ciertas comunas de las regiones de Coquimbo y Valparaíso. 
forestal) (Conaf, 2016a: 134-146). Pero no considera de manera directa la situación de personas dedicadas a la agricultura que se ven en la necesidad de emigrar y, por tanto, no incorpora medidas especiales para dichas consecuencias, considerando que dentro de los impactos socioambientales identificados se encuentra la emigración de sectores rurales a zonas urbanas.

Por su parte, el proyecto de Ley Marco de Cambio Climático no considera la situación de los desplazamientos internos dentro de las materias que regula, sin embargo, existen ciertas disposiciones aplicables a esta problemática que podrían constituir una posibilidad para abordar el tema; por ejemplo:

- Dentro del objeto de la Ley, se encuentra aumentar la resiliencia a los efectos adversos del cambio climático.

- Principio «costo-efectividad», que prioriza medidas que sean más efectivas para mitigar impactos al menor costo social, económico y ambiental posible.

- Principio de equidad: deber del Estado de procurar una justa asignación de cargas, costos y beneficios, con especial énfasis en sectores, comunidades y ecosistemas vulnerables.

- Concepto de adaptación al cambio climático y efectos adversos del cambio climático.

- Gestión del cambio climático, que se refiere al conjunto de eventuales políticas, planes, programas, normas, instrumentos, medidas y/o actividades destinadas a la mitigación y adaptación.

- Estrategia climática de largo plazo, que debe incluir directrices en materia de evaluación de riesgos asociados al cambio climático, considerando la vulnerabilidad de cada sector específico a sus efectos adversos.

\section{Respuesta institucional en México}

Según la revisión de antecedentes realizada, no existe una política pública o una regulación específica que aborde la migración interna por motivos agrícolas. Lo más parecido es el Programa de Jornaleras Agrícolas a cargo de Sedesol, que persigue mejorar las condiciones de vida de las personas ligadas a la agricultura que migran de manera temporal o permanente dentro del país. Este programa, otorga apoyos económicos directos (al arribo por hasta tres ocasiones al año por hogar), servicios de promoción social (dinero para alimentos de niñas y niños), subsidios para servicios básicos (estímulos para asistencia y permanencia escolar, atención a la salud, entre otros) y vivienda (alojamiento temporal, atención, información, espacios para estancia temporal) (Sedesol, 2019). 
No obstante, dicho programa ha sido calificado como asistencialista, dado que no resuelve el problema a largo plazo que viven las mujeres migrantes agrícolas (Tlanichollan, 2013: 97 y 99). Adicionalmente, existen otros programas del mismo tipo que persiguen atender aspectos relacionados con la migración, como el Sistema Nacional para el Desarrollo Integral de la Familia, el Programa de Educación Primaria para Niños y Niñas Migrantes, la Modalidad de Educación Multicultural para la Población Infantil Migrante, el Programa Vete Sano y Regresa Sano y el Programa de Movilidad Laboral Interna (Tlanichollan, 2013: 98). Estos programas no abordan la problemática migratoria desde un punto de vista preventivo, sino atienden las necesidades más básicas de la población desplazada.

Asimismo, cabe destacar que, a diferencia de lo que ocurre en Chile, en México se registran cifras relacionadas con la migración desde el primer censo que hizo el país en 1895 (Conapo, 2015: 30). Entre los datos considerados, se mide a aquellos residentes que se encuentran en una entidad federativa distinta a la de nacimiento, dato que permite conocer la migración interna acumulada. Adicionalmente, registra la población que no reside en su entidad federativa natal por lugar de nacimiento: este dato expresa a las entidades federativas que concentran emigrantes de su tierra de origen.

Adicionalmente, se realiza una Encuesta Nacional de la Dinámica Demográfica (Conapo, 2015a: 25), la que en el año 2014 determinó que la segunda causa que motiva la migración es el trabajo $(29,1 \%)$ y la segunda es la familia. Cifras similares muestran la migración de hace cinco años: los motivos laborales ocupan un 27,3\%. Estas encuestas permiten conocer información relacionada al fenómeno migratorio, que constituiría el fundamento para establecer regulaciones o políticas que aborden adecuadamente del asunto.

Ahora bien, México cuenta desde 2013, luego de la gran sequía, con una Política Pública Nacional para la Sequía que se concreta a través del Programa Nacional contra la Sequía (Pronacose), a cargo de Conagua, aunque participan muchos otros organismos estatales en su aplicación (Ortega-Gaucín, 2018: 93). El objetivo del Pronacose es elaborar programas de medidas para prevenir y enfrentar la sequía a nivel de cuenca o grupos de cuenca, desarrollar capacidad institucional local y, al mismo tiempo, coordinar y ejecutar acciones para reducir la vulnerabilidad de la población y mitigar las sequías existentes. Además, reconoce la afectación de la producción agrícola. Asimismo, contempla un concepto de sequía y la clasifica en estándares de intensidad desde anormalmente seco a sequía excepcional, estableciendo monitores de sequía. Este último resultaría más acorde para medir el fenómeno multifactorial de la sequía, dado que considera tres índices: precipitación estandarizada, escurrimiento y humedad de suelo, en conjunto con un mapa de persistencia de la sequía. Este indicador también determina la magnitud de la sequía en relación con la duración del fenómeno, que puede ser de uno a tres meses, hasta 12 meses o más. 
La Política Pública Nacional para la Sequía considera tanto acciones preventivas como de mitigación. Distingue aquellas que aplican antes del fenómeno y aquellas que aplican durante el mismo. No obstante, el foco principal se centra en la prevención; para ello Pronacose contempla líneas de acción: vulnerabilidad y evaluación de impactos y mitigación, preparación y respuesta. De este modo, busca detectar tempranamente la sequía, para lo cual se creó el Protocolo de Alerta Temprana y Acciones para Sequías, el que establece declaratorias de inicio de sequía por cuencas.

Finalmente, e indirectamente relacionado con la migración agrícola, es posible mencionar el Plan Nacional Hídrico, que tiene objetivos vinculados a la gestión integrada y sustentable del agua, como seguridad hídrica relacionada con la sequía; fortalecimiento del acceso al recurso hídrico y a los servicios sanitarios; incremento de las capacidades científicas, técnicas y tecnológicas del sector; y asegurar el agua para el riego agrícola, entre otras actividades, de manera sostenible. En particular respecto a la seguridad hídrica frente a las sequías se considera como una estrategia reducir la vulnerabilidad a los efectos del cambio climático (Conagua, 2014).

\section{Conclusiones}

La migración interna es un fenómeno que no ha sido lo suficientemente abordado en ninguno de los dos países analizados; dicha falta se observa tanto desde una perspectiva institucional, como jurídica. En esta línea, resulta evidente que el tema de este artículo - la migración de personas vinculadas a la agricultura en zonas ruralestampoco ha recibido un tratamiento adecuado desde las perspectivas señaladas.

En lo que refiere a una perspectiva institucional, el fenómeno de migración interna se puede deber a diversos motivos, como, por ejemplo, a los cambios climáticos, y dentro de los cuales se puede encontrar la falta de recursos hídricos para la subsistencia o desarrollo de actividades económicas, fenómeno que debiese ser considerado como factor de análisis en la elaboración de políticas públicas que pretendan abordar este tipo de procesos migratorios.

Si bien la migración agrícola es una estrategia adaptativa al cambio climático, debería acompañarse de respuestas institucionales que abarquen su complejidad; no solo encargándose de resolver aspectos inmediatos de la vida (respuesta reactiva), como ocurre en México, sino que explorando respuestas de tipo preventivas, como mejorar la gestión de recursos hídricos o elaborar mapas de riesgos a nivel nacional que señalen los lugares con mayor propensión a la migración por riesgos de sequía (como los decretos de escasez hídrica en Chile), con un particular enfoque en la agricultura (especialmente la de subsistencia), debido a la vulnerabilidad social que suele caracterizar a dicha población.

Por otra parte, cuando no se reconoce institucionalmente la migración interna, como en el caso de Chile, resulta necesario medir y observar el fenómeno, para luego 
promover las medidas preventivas y reactivas que sean necesarias. Esto debe estar acompañado de una metodología que considere indicadores para que, en el mediano o largo plazo, sea posible verificar su trazabilidad y eventualmente introducir mejoras. En este sentido, cabe mencionar como ejemplo, en el caso mexicano, la realización de estudios asociados a violencia y discriminación hacia mujeres migrantes, situación que permite elaborar medidas destinadas a abordar dicha circunstancia; una iniciativa que en Chile, y a la fecha, ni siquiera ha sido parte de algún levantamiento de información o estudio en general.

En este orden de ideas, la medición y observación del fenómeno migratorio también permitiría la implementación de medidas adaptativas, por ejemplo, para el caso de la agricultura: se puede estudiar la posibilidad de modificar las especies vegetales utilizadas y optar por aquellas que resistan de mejor manera los efectos derivados del cambio climático.

Es importante destacar que los casos analizados hablan más bien de una migración forzada y no voluntaria, como una medida de adaptación o incluso de sobrevivencia. En este sentido, las políticas públicas deberían diseñarse desde la perspectiva de la adaptabilidad al cambio climático y, de esta forma, propender a la educación y capacitación de personas dedicadas a la agricultura en lo que se refiere a cultivos sustentables, eficiencia en el uso de recursos hídricos, etcétera.

También, se observa que las personas propensas a migrar corresponden a mujeres, hombres e infantes, de manera que las políticas públicas que aborden esta temática debieran considerar enfoques de género, bienestar infantil y protección de la familia, así como identidad y arraigo, dado que la situación de los migrantes varía según género y conlleva consecuencias mayores cuando producto de la migración se afecta la unidad familiar y/o se separan hijos o hijas de sus padres o madres.

En lo que refiere a una perspectiva jurídica, tanto en el estatuto regulatorio de Chile como de México se observa la necesidad de considerar a la persona humana como un sujeto de protección, en el sentido de establecer derechos a su favor y deberes para los estados ante la eventualidad de encontrarse con personas en la necesidad de migrar de su lugar habitual de residencia o trabajo como consecuencia de cambios ambientales. En este sentido, las personas ligadas a actividades primarias, como la agricultura, son susceptibles a vivir este tipo de situaciones, razón por la cual requerirían de un tratamiento regulatorio particular que los ampare ante estos eventos, situación que no fue posible observar en ninguno de los casos analizados.

En relación con el estatuto jurídico de las aguas, si bien en México se ha consagrado el derecho humano al agua en su Constitución, en Chile aún existen aspectos pendientes. A nuestro juicio, es necesario consagrar el derecho humano al agua y establecer medidas que, ante situaciones de excepción, como la sequía, aseguren el acceso igualitario al recurso y consideren dentro de sus principios la sustentabilidad y la adaptabilidad al cambio climático. 
En este contexto, una ley general en Chile que regule en términos amplios las consecuencias derivadas del cambio climático, como la promoción de medidas de mitigación y adaptación al respecto, se hace urgente. Así, cabe mencionar que la situación de nuestro sujeto de estudio no es contemplada en el proyecto de ley recientemente presentado ante el Congreso, razón por la cual aún no es posible concluir que se esté avanzando en el tema. Por su parte, la ley mexicana considera la situación de los migrantes asociados a cambios en el clima y establece el deber de las entidades gubernamentales de prevenir y atender dicho fenómeno.

\section{Referencias}

Agua Para Todos (2020). «Iniciativa ciudadana de la Ley General de Aguas», Consejo Consultivo del Agua. Disponible en http://bit.ly/3nOw4GK.

Ángeles, Manuel y Alba Gámez (2013). «Eventos extremos, cambio climático y vulnerabilidad en México y Baja California Sur». En Gian Carlo Delgado, Carlos Gay, Mireya Imaz, María Amparo Martínez (coord.), México frente al cambio climático: retos y oportunidades (pp. 35-50). México: Centro de Investigaciones Interdisciplinarias en Ciencias y Humanidades Universidad Nacional Autónoma de México. Disponible en https://bit.ly/38yEifN.

BAEzA, Eduardo (2018). Sequía y escasez hídrica: Conceptos relacionados, situación actual y experiencia comparada en varios países para abordar el problema. Biblioteca del Congreso Nacional de Chile. Disponible en https://bit.ly/3ozeXBB. CR2, Centro de Ciencia del Clima y la Resiliencia (2015). La megasequía 2010-2015: Una lección para el futuro. Disponible en https://bit.ly/3plwrZL.

CESOP, Centro de Estudios Sociales y Opinión Pública (2019). «Jornaleros agrícolas migrantes y seguridad social». Disponible en https://bit.ly/3pAtip1. Conagua, Comisión Nacional del Agua (2014). Plan Nacional de Desarrollo 2013-2018: Programa Nacional Hídrico. Disponible https://bit.ly/38BDEoW.

Coneval, Consejo Nacional de Evaluación de la Política de Desarrollo Social (2018). Diez años de medición de pobreza multidimensional en México: avances y desafíos en política social. Disponible en https://bit.ly/2KWIe1C.

Conapo, Consejo Nacional de Población (2015a). Encuesta Nacional de la Dinámica Demográfica Enadid 2014. Disponible en https://bit.ly/2KTnbgI.

- (2015b). Prontuario de migración y movilidad interna. Disponible en http://bit. ly/2KoJqeB.

Conaf, Corporación Nacional Forestal (2016a). Estrategia Nacional de Cambio Climático y Recursos Vegetacionales 2017-2025. Disponible en https://bit.ly/3he63hE.

-. (2016b). Programa de Acción Nacional de Lucha Contra la Desertificación, la Degradación de Tierras y la Sequía. PANCD-Chile 2016-2030. Disponible en http:// bit.ly/3heiCJK. 
FAO, Organización de las Naciones Unidas para la Alimentación y la Agricultura (2010a). Gestión del riesgo de sequía y otros eventos climáticos extremos en Chile. Informe Región de O'Higgins. Disponible en http://www.fao.org/3/as39os/as39os.pdf.

-. (2010b) Gestión del riesgo de sequía y otros eventos climáticos extremos en Chile. Informe Región de Coquimbo. Disponible en http://www.fao.org/3/as391s/as391s.pdf.

-. (2016). Increasing the resilience of agricultural livelihoods. Disponible en http:// www.fao.org/3/a-i5615e.pdf.

Gamboa Claudia y Gutiérrez Miriam, Cámara de Diputados LXIII Legislatura Dirección de Servicios de Investigación y Análisis, (2015). «Jornaleros Agrícolas en México: Antecedentes, Políticas Públicas, Tratados Internacionales, Causas y Efectos del Problema, Iniciativas y Opiniones Especializadas». Disponible en https://bit.ly/3pyIrY2.

Granados Alcantar, José y María Quezada Ramírez (2018). «Tendencias de la migración interna de la población indígena en México 1995 a 2015». Estudios Demográficos y Urbanos, 2: 327-363. Disponible en http://bit.ly/3hAEU8P.

Guillof, Matías (2013). «El derecho al agua como un derecho humano: El caso de la Provincia de Petorca». En Tomas Vial (coord.), Informe anual de derechos humanos (pp. 255-282). Santiago: Ediciones UDP. Disponible en https://bit.ly/3aGoJ8I.

Gutiérrez Oliva, Andrés (2019). «Escasez hídrica en Chile. Desafíos para el consumo humano y perspectiva en modelos comparados». Santiago: Fundación Newenko. Disponible en https://bit.ly/3aDbup1.

Hoegh-Guldberg, O., D. Jacob, M. Taylor, M. Bindi, S. Brown (2018). «Impacts of $1.5^{\circ} \mathrm{C}$ Global Warming on Natural and Human Systems». En IPCC, Global Warming of $1.5^{\circ} \mathrm{C}$. Disponible en https://bit.ly/3n6XXsI.Inecc, Instituto Nacional de Ecología y Cambio Climático (2016). «Vulnerabilidad al cambio climático». Disponible en http://bit.ly/3nMdxeg.

INEGI, Instituto Nacional de Estadística y Geografía (2010). Censo de Población y Vivienda 2010. Disponible en https://bit.ly/3mKL5bl.

-. (2011). Panorama sociodemográfico de Guerrero. Disponible en http://bit. ly/2KWXwDB.

LARRAín Ruiz-TAgLE, Sara (2006). «El agua en Chile: entre los derechos humanos y las reglas de mercado». Polis. Revista Latinoamericana, 14. Disponible en http:// bit.ly/3mMck5p.

Ministerio de Desarrollo Social, Chile (2018). Informe de Desarrollo Social. Disponible en https://bit.ly/38xSENx.

Mishra, Ashok y Vijay Singh (2010). «A review of drought concepts». Journal of Hydrology, 391 (1-2): 202-216. Disponible en http://bit.ly/2KOhi4i.

Morales Jiménez, J., A. Bustamante González, S. Vargas López y U. Cano Castañeda (2018). "Agricultura y migración Colectiva en La Región Montaña, México», Agroproductividad, 11 (10): 87-92. Disponible en http://bit.ly/30oz1. 
Naciones Unidas, Asamblea General, Convención Marco de las Naciones Unidas sobre Cambio Climático (CMNUCC), 1992, disponible en: https://www.acnur. org/fileadmin/Documentos/BDL/2009/6907.pdf

Noralene, Uy, Rajib Shaw y Yukiko Takeuchi (2012). «Linking Livelihoods and Ecosystems for Enhance Disaster». En Rjaib Shaw y Phong Tran (eds.), Management. Environment Disaster Linkages, Community, Environment and Disaster Risk Management (pp. 131-145). Emerald Books.

OIM, Organización Internacional para las Migraciones (2014). Glosario Migración Medio Ambiente y Cambio Climático datos empíricos para la formulación de políticas. Disponible en https://bit.ly/3pBsPDo.

-. (2017). Migraciones, ambiente y cambio climático. Estudios de casos en América del sur. Cuadernos Migratorios 8. Buenos Aires. Disponible en https://bit. ly/3rosANr.

-. (2018). Global Migrations Indicators. Berlín. Disponible en https://bit.ly/3hh4Wha.

-. (2019). La movilidad humana en la agenda climática de las américas: necesidades y oportunidades. San José. Disponible en http://bit.ly/37LkOFs.

Ortega-Gaucín, David (2018). «Medidas para afrontar la sequía en México: una visión retrospectiva». Revista del Colegio de San Luis, 8 (15): 77-105. Disponible en https://bit.ly/3hs5u49.

Sedesol, Secretaría de Desarrollo Social (2019). Programa de Atención a Jornaleros Agrícolas. Disponible en https://bit.ly/2M5eS1U.

Tlanichollan, Centro de Derechos Humanos La Montaña (2013). La Montaña de Guerrero: Tierra de Mujeres Migrantes. Disponible en https://bit.ly/38wSVQN.

Vergara, Alejandro (2011). «Sistema y autonomía del derecho de aguas». En Actas de Derecho de Aguas núm. 1 (pp. 57-78). Santiago: Abeledo Perrot y Legal Publishing WWF, World Wildlife Fund (2010). Impactos y vulnerabilidad al Cambio Climático en México. Disponible en https://bit.ly/3mNHo6n.

\section{Sobre los autores}

Gustavo Arellano Reyes es abogado de la Universidad de Chile. Actualmente trabaja en la Fiscalía de la Superintendencia del Medio Ambiente. Antes se desempeñó como abogado en la Dirección Ejecutiva del Servicio de Evaluación Ambiental. Su correo electrónico es gustavoarellanoreyes@gmail.com. (D) http://orcid. org/0000-0002-8749-3949.

Carolina Silva Santelices es abogada de la Universidad de Chile. Maestra en Razonamiento Probatorio en las universidades de Girona y de Génova, y maestra en Ciencias Ambientales de la Universidad Autónoma de San Luis Potosí, México y la University of Applied Sciences Colonia, Alemania. Su correo electrónico es carolinasilveli@gmail.com. (D) http://orcid.org/0000-0001-8399-2540. 
La Revista de Derecho Ambiental, del Centro de Derecho Ambiental de la Facultad de Derecho de la Universidad de Chile, es un espacio de exposición y análisis en el plano académico del derecho ambiental. Su contenido se presenta a través de doctrina, jurisprudencia y recensiones, y aborda diversas materias relacionadas con la gestión, institucionalidad y herramientas de protección ambiental y desarrollo sustentable. Se presentan artículos de diferentes autores y autoras en los que se analizan y abordan casos y temas jurídico-ambientales de creciente interés y actualidad.

\author{
DIRECTORA \\ Valentina Durán Medina \\ EDITORES \\ Jorge Ossandón Rosales \\ y Antonio Pulgar Martínez \\ SITIO WEB \\ revistaderechoambiental.uchile.cl \\ CORREO ELECTRÓNICO \\ revistada@derecho.uchile.cl \\ LICENCIA DE ESTE ARTÍ́CULO \\ Creative Commons Atribución Compartir Igual 4.o Internacional
}

La edición de textos, el diseño editorial

y la conversión a formatos electrónicos de este artículo

estuvieron a cargo de Tipográfica

(www.tipografica.io) 